\title{
TITLE:
}

\section{Aligning single-wall carbon nanotubes with an alternating- current electric field}

\section{AUTHOR(S):}

Chen, XQ; Saito, T; Yamada, H; Matsushige, K

\section{CITATION:}

Chen, XQ ...[et al]. Aligning single-wall carbon nanotubes with an alternating-current electric field. APPLIED PHYSICS LETTERS 2001, 78(23): 3714-3716

\section{ISSUE DATE:}

2001-06-04

URL:

http://hdl.handle.net/2433/39645

\section{RIGHT:}

Copyright 2001 American Institute of Physics. This article may be downloaded for personal use only. Any other use requires prior permission of the author and the American Institute of Physics. 


\title{
Aligning single-wall carbon nanotubes with an alternating-current electric field
}

\author{
X. Q. Chen ${ }^{\mathrm{a})}$ and T. Saito \\ Venture Business Laboratory, Kyoto University, Kyoto 606-8501, Japan \\ H. Yamada and K. Matsushige \\ Department of Electronic Science and Engineering, Kyoto University, Kyoto 606-8501, Japan
}

(Received 5 March 2001; accepted for publication 12 April 2001)

\begin{abstract}
Single-wall carbon nanotubes (SWCNTs) were highly aligned by an external electric field. The results suggest that the alignment of SWCNTs shows significant dependencies on the frequency and the magnitude of the applied electric field. The electric field with $5 \mathrm{MHz}$ straightened out the SWCNTs and created highly oriented samples with fewer large particles. We also discussed the mechanism and applications. (c) 2001 American Institute of Physics. [DOI: 10.1063/1.1377627]
\end{abstract}

Since their discovery, ${ }^{1}$ carbon nanotubes $(\mathrm{CNTs})$ have attracted strong attention because of their anisotropic structure and outstanding electrical and mechanical properties. ${ }^{2}$ Individual CNTs have been utilized to fabricate molecular devices such as field-effect transistors ${ }^{3,4}$ and to measure their mechanical properties. ${ }^{5}$ Although carbon nanotubes could be manipulated by using an atomic-force microscope (AFM) tip, ${ }^{6}$ the efficiency was very low; the tip has also probably caused much damage to the structure of the carbon nanotubes. Therefore, it is still difficult to handle or align individual CNTs to ideal locations. On the other hand, aligned CNT films would offer many advantages compared to random tangled deposited bulk samples. Several aligning methods have been demonstrated. Zhang and Iijima ${ }^{7}$ aligned single-wall carbon nanotubes (SWCNTs) through introducing flowing argon gas in the laser ablation reactor. Smith et al. ${ }^{8}$ produced thick films of aligned SWCNTs and ropes by filtration/deposition from suspension in strong magnetic fields. They showed the macroscopic oriented arrays of SWCNTs. Yamamoto, Akita, and Nakayama, ${ }^{9,10}$ oriented multiwall carbon nanotubes (MWCNTs) using electrophoresis, and then Bubke et al. ${ }^{11}$ also aligned MWCNTs by an electric field and measured the optical anisotropy. We find that alignment by electric field is a simple and efficient method. In this letter, we report on the macroscopic alignment of SWCNTs by an electric field. The dependencies of this alignment on the frequency and the magnitude of the electric field are also described and discussed.

The SWCNTs, which were fabricated by the arcdischarge method, were commercially obtained from BuckyUSA. The nanotubes were ultrasonically dispersed in ethanol and this suspension was centrifuged to remove large particles. Gold interdigitated electrodes [schematically shown in Fig. 1(a)] were prepared on $\mathrm{Si} / \mathrm{SiO}_{2}$ substrates by the vacuum deposition method. The gap between two electrodes is 25 $\mu \mathrm{m}$ and the thickness of the electrodes is $50 \mathrm{~nm}$. The substrates were immersed in the SWCNT suspension and an

\footnotetext{
a) Author to whom correspondence should be addressed; electronic mail: xchen@northwestern.edu
}

electric field was applied to the electrodes for $15 \mathrm{~min}$. Then, the samples were taken out and dried in air.

Figure 1(b) shows the scanning electron microscope (SEM) image of the SWCNT samples aligned by applying an

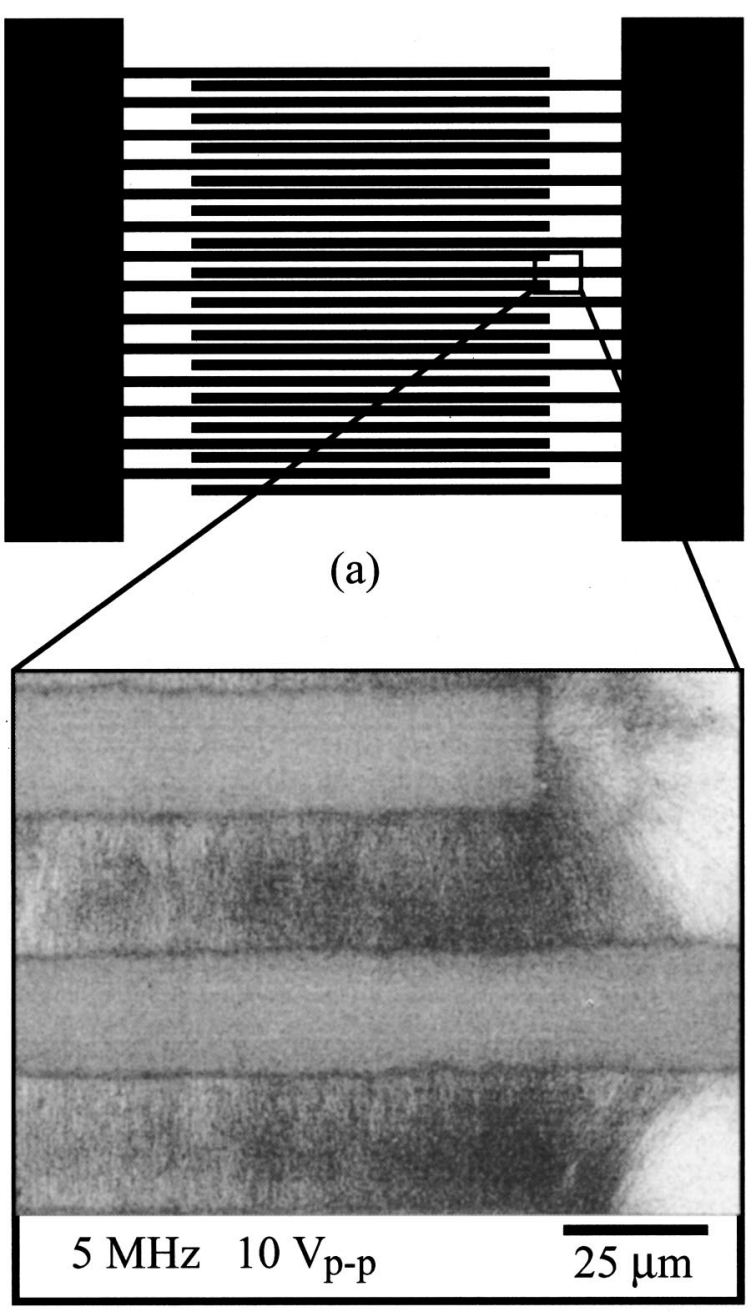

(b)

FIG. 1. (a) Schematic diagram of gold electrode patterns prepared on $\mathrm{Si} / \mathrm{SiO}_{2}$ substrates. (b) SEM image of the SWCNT sample aligned with an ac electric field. 

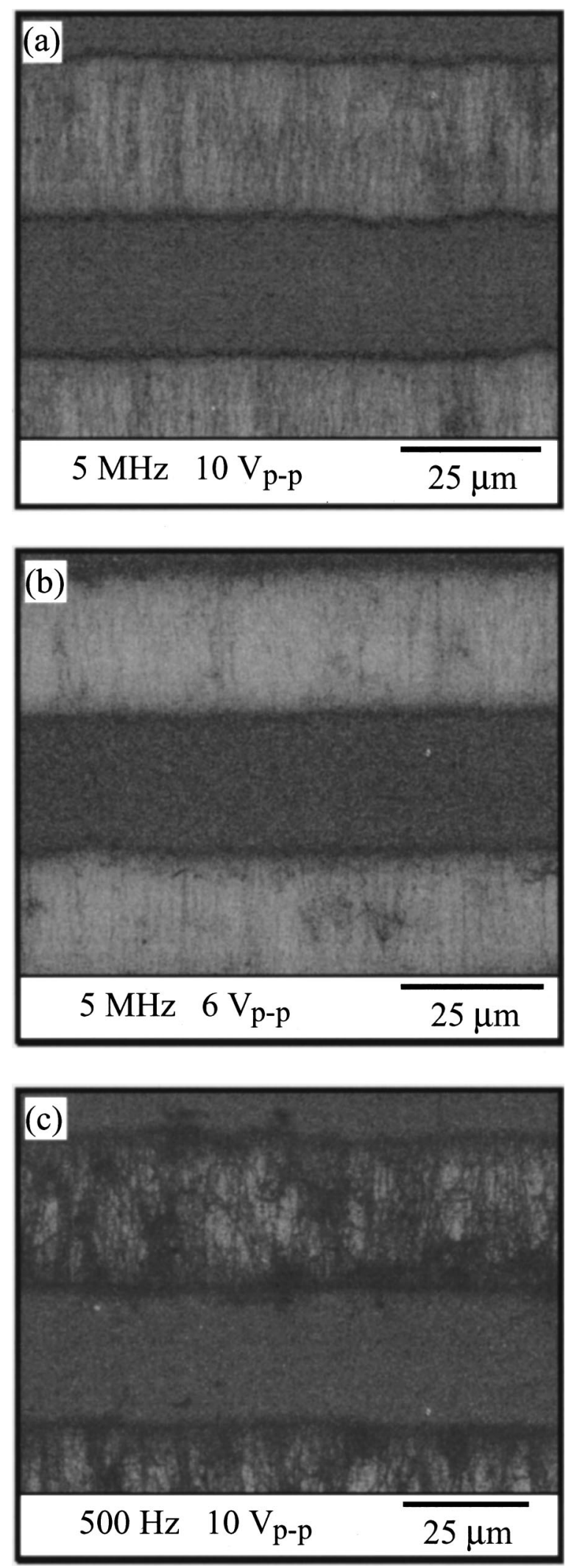

FIG. 2. SEM images of the SWCNT samples aligned in different conditions. (a) Aligned by applying an ac electric field with a frequency of 5 $\mathrm{MHz}$ and a voltage of $10 \mathrm{~V}$ peak to peak. (b) Aligned by applying an ac electric field with a frequency of $5 \mathrm{MHz}$ and a voltage of $6 \mathrm{~V}$ peak to peak. (c) Aligned by applying an ac electric field with a frequency of 500 $\mathrm{Hz}$ and a voltage of $10 \mathrm{~V}$ peak to peak.

ac electric field with a frequency of $5 \mathrm{MHz}$ and a magnitude of $10 \mathrm{~V}$ peak to peak. It can be observed that the SWCNTs were highly aligned along the direction of the electric flux. Downloaded 30 May 2007 to 130.54.110.22. Redistribution subjec
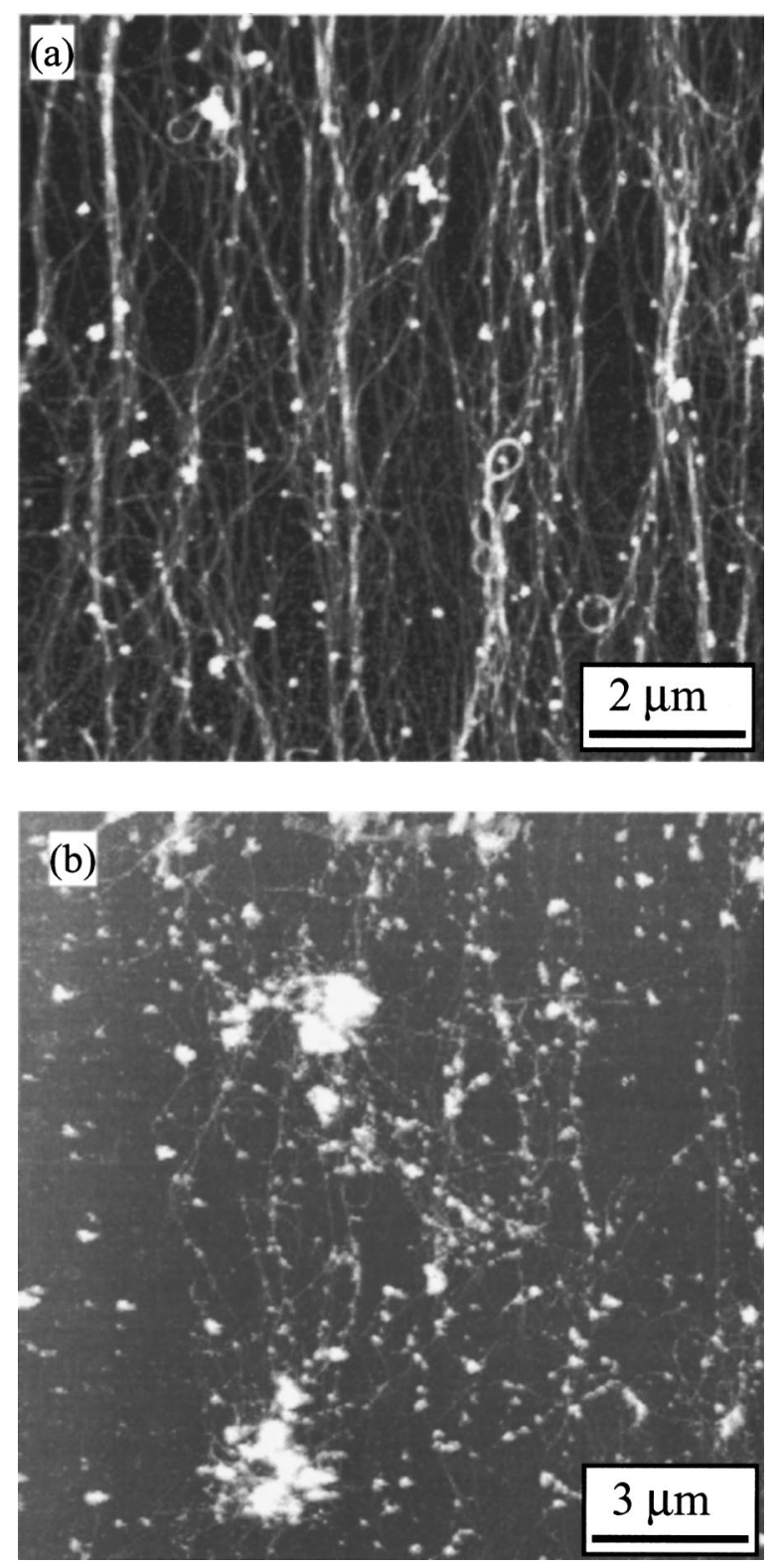

FIG. 3. Tapping-mode AFM images of the aligned SWCNT samples. (a) Aligned by applying an ac electric field with a frequency of $5 \mathrm{MHz}$ and a voltage of $10 \mathrm{~V}$ peak to peak. (b) Aligned by applying an ac electric field with a frequency of $500 \mathrm{~Hz}$ and a voltage of $10 \mathrm{~V}$ peak to peak.

The concentration of SWCNTs at the angles of the electrodes is obviously higher than that at the central area of the gaps. AFM topographic images (data not shown) show that most of the carbon nanotubes have a length of several microns. However, the image demonstrates that the SWCNTs were aligned and linked up to the electrodes. Therefore, the result suggests that many carbon nanotubes have overlapped between two electrodes and formed long ropes.

We also find that the nanotube orientation is strongly dependent on the magnitude and the frequency of the electric field. Figure 2 illustrates the SEM images of the samples aligned with the electric field with different magnitudes and frequencies. The sample aligned by the electric field with a frequency of $5 \mathrm{MHz}$ and a magnitude of $10 \mathrm{~V}$ peak to peak is shown in Fig. 2(a), and the sample aligned by the electric field with a frequency of $5 \mathrm{MHz}$ and a magnitude of $6 \mathrm{~V}$ peak to peak is shown in Fig. 2(b), and Fig. 2(c) demonstrates the sample aligned by the electric field with a fre-
o AlP license or copyright, see http://apl.aip.org/apl/copyright.jsp 
quency of $500 \mathrm{~Hz}$ and a magnitude of $10 \mathrm{~V}$ peak to peak. By comparing Figs. 2(a) and 2(b), we can find that the concentration of the aligned SWCNTs is dependent on the magnitude of the electric field. When the magnitude of the electric field was decreased, there were fewer carbon nanotubes attracted in the gaps. This suggests that the electrostatic force determines the aligning speed.

In comparison with Fig. 2(c), the result shows that the degree of the alignment is apparently depending on the frequency of the ac electric field. When the frequency of the electric field was decreased to $500 \mathrm{~Hz}$, there were many aggregations of the SWCNTs existing within the electrode gaps. We also applied the electric field with frequencies of 500,50 , and $5 \mathrm{kHz}$, and dc voltage to align the SWCNTs. The results demonstrate that the alignment degree of the carbon nanotubes was reduced gradually with the decrease of the frequency of the electric field. There was no obvious alignment in the samples applied by the dc electric field.

The aligning experiment of the SWCNTs described above is highly reproducible. For this alignment, the results could be rationalized by considering the electronic polarization induced in carbon nanotubes by the electric field. Because of the structure anisotropy of the carbon nanotubes, the dipole moment in the direction parallel to the tube axis is quite stronger than that in the perpendicular direction. Therefore, carbon nanotubes could be aligned along the direction of the electric field if the movement is not restricted by its environment.

Although the SWCNT samples oriented by the dc electric field did not demonstrate the apparent orientation, there were many tangled nanotubes driven to the anode by the electrostatic force. This is different from a previous report, ${ }^{9}$ which reported that the MWCNTs moved towards the cathode under the influence of the electric field. Based on the above alignment mechanism, it seems to be reasonable that carbon nanotubes with a perfect structure will move towards both the anode and the cathode because of the concentration of the electric field at the edges of the electrodes. The selective deposition of the carbon nanotubes is probably attributed to the structure defects or the charge of the carbon nanotubes, which could be made in the sample preparing process. The SWCNTs used in this work were aggregated on the anode when the dc electric field was applied, which indicates that the nanotubes were negatively charged. We measured the surface potential of the SWCNTs on a graphite substrate by Kelvin-force microscopy. The result demonstrates that the nanotubes have negative potential.
The above results also show that the frequency of the electric field has a strong influence on the orientation of carbon nanotubes. In the sample aligned by an electric field with a low frequency, there were many tangled nanotubes (see Fig. 2), which are often found in samples prepared by the casting method. This implies that there are many such tangled nanotubes in the suspension. It seemed that the electric field with a high frequency straightened out the nanotubes.

Figure 3 illustrates the AFM images of the aligned SWCNT samples. The sample aligned by the electric field with a frequency of $5 \mathrm{MHz}$ and a voltage of $10 \mathrm{~V}$ peak to peak is shown in Fig. 3(a). Figure 3(b) demonstrates the sample aligned by the electric field with a frequency of 500 $\mathrm{Hz}$ and a voltage of $10 \mathrm{~V}$ peak to peak. It has been observed that the higher the frequency of the electric field the fewer the large particles in the samples. Therefore, one of the applications of this alignment may be the purification of raw SWCNTs. In addition, ropes of SWCNTs with a length of more than $10 \mu \mathrm{m}$ have also been formed in highly oriented samples.

In summary, we aligned SWCNTs by applying an ac electric field. Highly oriented SWCNT samples were prepared when an electric field with a frequency of $5 \mathrm{MHz}$ was applied. The alignment of the SWCNTs demonstrates significant dependencies on the frequency and the magnitude of the electric field. The mechanism and the applications of the alignment were also discussed, though investigation of them is still necessary. The method will have potential application in handling nanotubes.

${ }^{1}$ S. Iijima, Nature (London) 354, 56 (1991).

${ }^{2}$ R. Saito, G. Dresselhaus, and M. S. Dresselhaus, Physics Properties of Carbon Nanotubes (Imperial College, London, U.K. 1998).

${ }^{3}$ S. J. Tans, A. R. M. Verschueren, and C. Dekker, Nature (London) 393, 49 (1998).

${ }^{4}$ R. Martel, T. Schmidt, H. R. Shea, T. Hertel, and Ph. Avouris, Appl. Phys. Lett. 73, 2447 (1998).

${ }^{5}$ M. Yu, B. S. Files, S. Arepalli, and R. S. Ruoff, Phys. Rev. Lett. 84, 5552 (2000).

${ }^{6}$ T. Hertel, R. Martel, and Ph. Avouris, J. Phys. Chem. B 102, 910 (1998).

${ }^{7}$ Y. Zhang and S. Iijima, Phys. Rev. Lett. 82, 3472 (1999).

${ }^{8}$ B. W. Smith, Z. Benes, D. E. Luzzi, J. E. Fischer, D. A. Walters, M. J. Casavant, J. Schmidt, and R. M. Smalley, Appl. Phys. Lett. 77, 663 (2000).

${ }^{9}$ K. Yamamoto, S. Akita, and Y. Nakayama, Jpn. J. Appl. Phys., Part 2 35, L917 (1996).

${ }^{10}$ K. Yamamoto, S. Akita, and Y. Nakayama, J. Phys. D 31, L34 (1998).

${ }^{11}$ K. Bubke, H. Gnewuch, M. Hempstead, J. Hammer, and L. H. Green, Appl. Phys. Lett. 71, 1906 (1997). 